\title{
Chemotherapie des Mamma-Karzinoms
}

Erfahrungen in der Behandlung des metastasierten Mamma-Karzinoms, some 5-Jahres-Ergebnisse zur adjuvanten Therapie mit 5-Fluorouracil, Adriamycin und Cyclophosphamid

\begin{tabular}{|l|l|l|}
\hline S. & Sewa & Legha \\
\hline
\end{tabular}

M.D., Anderson-Hospital and Tumor Institute, The University of Texas System Cancer Center, USA - Houston/Texas

Vortrag am 17. Februar 1981 in Nürnberg, Med. Klinik

Gesamt

19,5 Monate 16,0 Monate

32 Monate

24 Monate

17 Monate

5 Monate

Prof. Legha vom M.D. Anderson Hospital, Houston/Texas, Department for Breast Cancer, berichtete anläßlich eines Auf-enthaltes bei Herrn Prof. Gallmeier am 17. Februar 1981 in Nürnberg über die Erfahrungen zur Chemotherapie des fort-geschrittenen Mamma-Karzinoms mit der Kombination 5-Fu, Adriamycin und Cyclophosphamid (FAC-Schema).

In den Jahren 1973-1976 wurden 619 Patienten mit der Kombination aus

5-Fluorouracil

Adriamycin

Cyclophosphamid

behandelt.

500mg/m2i.v. Tl + Í 50 mg/m2 i. v. T 1 500mg/m2i.v. T1 + !

Die Auswertung der Therapie ergab folgende Ergebnisse:

Patientenzahl 619

Vollremission $18 \%$

Teilremission $47 \%$

Gesamt $\quad 65 \%$

Mediane Dauer der Remission:

Vollremissionen

Teilremissionen

Mediane Dauer der Überlebenszeiten:

Vollremissionen

Teilremissionen

Improvement

Patienten mit Progression

Eine zusätzliche Gabe von BCG brachte keine Verbesserung der Ergebnisse.

Aufgrund der guten Erfahrungen beim fortgeschrittenen Mamma-Karzinom (die Remissionsraten durch die ADM-hal-tigen Schemata lagen um 10-15 \% höher als die der verschie-denen CMF- 
Regimen und auch die ÜLZ, bzw. die Remis-sionsdauer war en 5-6 Monate länger) entschloß man sich, die Kombination aus

5-Fluorouracil

Adriamycin

Cyclophosphamid

$400 \mathrm{mg} / \mathrm{m} 2$ i. v. Tl + Í

$40 \mathrm{mg} / \mathrm{m} 2$ i.v. T $1400 \mathrm{mg} / \mathrm{m} 2$ i.v. T 1

auch adjuvant einzusetzen (Wiederholung alle 28 Tage). BCG wurde zusätzlich an Tag 9, 13 und 17 durch Skarifizie-rung angewandt.

Nach Erreichen einer Höchstdosis von 300 mg/m2 Adriamycin wurde Methotrexat anstatt Adriamycin eingesetzt. Die Therapiedauer betrug 2 Jahre. Prof. Legha berichtete über die 5Jahresergebnisse dieser Studie:

Gruppe 1:

N, N

Patienten $<50$ Jahre

$77 \%$ NED 69 \% NED

" $1-3$

$>4$

36 Patienten 8 Rezidive

13 Rezidive

22 Rezidive 43 Rezidive

$71 \%$ NED $72 \%$ NED

42 Patienten

75 Patienten 153 Patienten

Zusammenfassend beendet Prof. Legha sein Referat mit den Schlußfolgerungen:

Adriamycin ist die aktivste Monosubstanz in der Behandlung des Mamma-Karzinoms und erreicht eine Responsrate, die äquivalent ist mit der einiger Kombinationschemothera-pien.

ADM-haltige Kombinationstherapien erzielen eine höhere Responsrate, längere

Remissionszeiten und eine längere ÜLZ als andere nicht Adriamycin-haltige Chemotherapien. Aufgrund der Überlegenheit der ADM-haltigen Schemata beim fortgeschrittenen MammaKarzinom sind sie auch als adjuvante Therapie des Early-Breast-Cancer eingesetzt worden und erzielten eine signifikante Verlängerung des rezidivfreien Intervalls unabhängig vom Menopausen-Status.

Das Risiko der Kardiotoxizität bei einer Dosis bis 500 mg/m2 variiert zwischen 1-5 \% und ist abhängig von der Koexistenz anderer Risikofaktoren. Die Kardiotoxizität ist durch ein sorgfältiges Monitoring der Herzfunktion ver-meidbar. 